\title{
Measurement of $b$-hadron branching fractions for two-body decays into charmless charged hadrons
}

\section{The LHCb collaboration}

ABstract: Based on data corresponding to an integrated luminosity of $0.37 \mathrm{fb}^{-1}$ collected by the $\mathrm{LHCb}$ experiment in 2011, the following ratios of branching fractions are measured:

$$
\begin{aligned}
\mathcal{B}\left(B^{0} \rightarrow \pi^{+} \pi^{-}\right) / \mathcal{B}\left(B^{0} \rightarrow K^{+} \pi^{-}\right) & =0.262 \pm 0.009 \pm 0.017 \\
\left(f_{s} / f_{d}\right) \cdot \mathcal{B}\left(B_{s}^{0} \rightarrow K^{+} K^{-}\right) / \mathcal{B}\left(B^{0} \rightarrow K^{+} \pi^{-}\right) & =0.316 \pm 0.009 \pm 0.019 \\
\left(f_{s} / f_{d}\right) \cdot \mathcal{B}\left(B_{s}^{0} \rightarrow \pi^{+} K^{-}\right) / \mathcal{B}\left(B^{0} \rightarrow K^{+} \pi^{-}\right) & =0.074 \pm 0.006 \pm 0.006 \\
\left(f_{d} / f_{s}\right) \cdot \mathcal{B}\left(B^{0} \rightarrow K^{+} K^{-}\right) / \mathcal{B}\left(B_{s}^{0} \rightarrow K^{+} K^{-}\right) & =0.018_{-0.007}^{+0.008} \pm 0.009 \\
\left(f_{s} / f_{d}\right) \cdot \mathcal{B}\left(B_{s}^{0} \rightarrow \pi^{+} \pi^{-}\right) / \mathcal{B}\left(B^{0} \rightarrow \pi^{+} \pi^{-}\right) & =0.050_{-0.009}^{+0.011} \pm 0.004 \\
\mathcal{B}\left(\Lambda_{b}^{0} \rightarrow p \pi^{-}\right) / \mathcal{B}\left(\Lambda_{b}^{0} \rightarrow p K^{-}\right) & =0.86 \pm 0.08 \pm 0.05
\end{aligned}
$$

where the first uncertainties are statistical and the second systematic. Using the current world average of $\mathcal{B}\left(B^{0} \rightarrow K^{+} \pi^{-}\right)$and the ratio of the strange to light neutral $B$ meson production $f_{s} / f_{d}$ measured by LHCb, we obtain:

$$
\begin{aligned}
\mathcal{B}\left(B^{0} \rightarrow \pi^{+} \pi^{-}\right) & =(5.08 \pm 0.17 \pm 0.37) \times 10^{-6}, \\
\mathcal{B}\left(B_{s}^{0} \rightarrow K^{+} K^{-}\right) & =(23.0 \pm 0.7 \pm 2.3) \times 10^{-6}, \\
\mathcal{B}\left(B_{s}^{0} \rightarrow \pi^{+} K^{-}\right) & =(5.4 \pm 0.4 \pm 0.6) \times 10^{-6} \\
\mathcal{B}\left(B^{0} \rightarrow K^{+} K^{-}\right) & =\left(0.11_{-0.04}^{+0.05} \pm 0.06\right) \times 10^{-6} \\
\mathcal{B}\left(B_{s}^{0} \rightarrow \pi^{+} \pi^{-}\right) & =\left(0.95_{-0.17}^{+0.21} \pm 0.13\right) \times 10^{-6}
\end{aligned}
$$

The measurements of $\mathcal{B}\left(B_{s}^{0} \rightarrow K^{+} K^{-}\right), \mathcal{B}\left(B_{s}^{0} \rightarrow \pi^{+} K^{-}\right)$and $\mathcal{B}\left(B^{0} \rightarrow K^{+} K^{-}\right)$are the most precise to date. The decay mode $B_{s}^{0} \rightarrow \pi^{+} \pi^{-}$is observed for the first time with a significance of more than $5 \sigma$.

KEYWORDS: Hadron-Hadron Scattering

ARXIV EPRINT: 1206.2794 


\section{Contents}

1 Introduction 1

2 Detector, trigger and event selection $\quad 2$

3 Particle identification 3

4 Invariant mass fits to $H_{b} \rightarrow h^{+} h^{\prime-}$ spectra 9

5 Systematic uncertainties $\quad 11$

$6 \quad$ Results and conclusions 12

$\begin{array}{ll}\text { The LHCb collaboration } & 16\end{array}$

\section{Introduction}

In the quest for physics beyond the Standard Model (SM) in the flavour sector, the study of charmless $H_{b} \rightarrow h^{+} h^{-}$decays, where $H_{b}$ is a $b$-flavoured meson or baryon, and $h^{(\prime)}$ stands for a pion, kaon or proton, plays an important role. A simple interpretation of the $C P$-violating observables of the charmless two-body $b$-hadron decays in terms of CabibboKobayashi-Maskawa (CKM) weak phases $[1,2]$ is not possible. The presence of so-called penguin diagrams in addition to tree diagrams gives non-negligible contributions to the decay amplitude and introduces unknown hadronic factors. This then poses theoretical challenges for an accurate determination of CKM phases. On the other hand, penguin diagrams may have contributions from physics beyond the SM [3-7]. These questions have motivated an experimental programme aimed at the measurement of the properties of these decays [8-12].

Using data corresponding to an integrated luminosity of $0.37 \mathrm{fb}^{-1}$ collected by the LHCb experiment in 2011, we report measurements of the branching fractions $\mathcal{B}$ of the $B^{0} \rightarrow \pi^{+} \pi^{-}, B_{s}^{0} \rightarrow K^{+} K^{-}, B_{s}^{0} \rightarrow \pi^{+} K^{-}, B^{0} \rightarrow K^{+} K^{-}$and $B_{s}^{0} \rightarrow \pi^{+} \pi^{-}$decays. Furthermore, we also measure the ratio of the $\Lambda_{b}^{0} \rightarrow p \pi^{-}$and $\Lambda_{b}^{0} \rightarrow p K^{-}$branching fractions. The inclusion of charge-conjugate decay modes is implied throughout the paper.

The ratio of branching fractions between any two of these decays can be expressed as

$$
\frac{\mathcal{B}\left(H_{b} \rightarrow F\right)}{\mathcal{B}\left(H_{b}^{\prime} \rightarrow F^{\prime}\right)}=\frac{f_{H_{b}^{\prime}}}{f_{H_{b}}} \cdot \frac{N\left(H_{b} \rightarrow F\right)}{N\left(H_{b}^{\prime} \rightarrow F^{\prime}\right)} \cdot \frac{\varepsilon_{\mathrm{rec}}\left(H_{b}^{\prime} \rightarrow F^{\prime}\right)}{\varepsilon_{\mathrm{rec}}\left(H_{b} \rightarrow F\right)} \cdot \frac{\varepsilon_{\mathrm{PID}}\left(F^{\prime}\right)}{\varepsilon_{\mathrm{PID}}(F)}
$$

where $f_{H_{b}^{(\prime)}}$ is the probability for a $b$ quark to hadronize into a $H_{b}^{(\prime)}$ hadron, $N$ is the observed yield of the given decay to the final state $F^{(\prime)}, \varepsilon_{\text {rec }}$ is the overall reconstruction 
efficiency, excluding particle identification (PID), and $\varepsilon_{\text {PID }}$ is the PID efficiency for the corresponding final state hypothesis. We choose to measure ratios where a better cancellation of systematic uncertainties can be achieved.

\section{Detector, trigger and event selection}

The LHCb detector [13] is a single-arm forward spectrometer covering the pseudorapidity range $2<\eta<5$, designed for the study of particles containing $b$ or $c$ quarks. The detector includes a high-precision tracking system consisting of a silicon-strip vertex detector surrounding the $p p$ interaction region, a large-area silicon-strip detector located upstream of a dipole magnet with a bending power of about $4 \mathrm{Tm}$, and three stations of silicon-strip detectors and straw drift-tubes placed downstream. The combined tracking system has momentum resolution $\Delta p / p$ that varies from $0.4 \%$ at $5 \mathrm{GeV} / c$ to $0.6 \%$ at $100 \mathrm{GeV} / c$, and impact parameter resolution of $20 \mu \mathrm{m}$ for tracks with high transverse momenta. Charged hadrons are identified using two ring-imaging Cherenkov (RICH) detectors. Photon, electron and hadron candidates are identified by a calorimeter system consisting of scintillating-pad and pre-shower detectors, an electromagnetic calorimeter and a hadronic calorimeter. Muons are identified by a muon system composed of alternating layers of iron and multiwire proportional chambers. The trigger consists of a hardware stage, based on information from the calorimeter and muon systems, followed by a software stage which performs a full event reconstruction.

The software trigger requires a two-, three- or four-track secondary vertex with a high sum of the transverse momenta of the tracks, significant displacement from the primary interaction, and at least one track with a transverse momentum exceeding $1.7 \mathrm{GeV} / c$. Furthermore, it exploits the impact parameter, defined as the smallest distance between the reconstructed trajectory of the particle and the $p p$ collision vertex, requiring its $\chi^{2}$ to be greater than 16. A multivariate algorithm is used for the identification of the secondary vertices [14]. In addition, a dedicated two-body software trigger is used. To discriminate between signal and background events, this trigger selection imposes requirements on: the quality of the online-reconstructed tracks $\left(\chi^{2} / \mathrm{ndf}\right.$, where ndf is the number of degrees of freedom), their transverse momenta $\left(p_{\mathrm{T}}\right)$ and their impact parameters $\left(d_{\mathrm{IP}}\right)$; the distance of closest approach of the daughter particles $\left(d_{\mathrm{CA}}\right)$; the transverse momentum of the $b$-hadron candidate $\left(p_{\mathrm{T}}^{B}\right)$, its impact parameter $\left(d_{\mathrm{IP}}^{B}\right)$ and its decay time $\left(t_{\pi \pi}\right.$, calculated assuming decay into $\pi^{+} \pi^{-}$). Only $b$-hadron candidates within the $\pi^{+} \pi^{-}$invariant mass range $4.7-$ $5.9 \mathrm{GeV} / c^{2}$ are accepted. The $\pi^{+} \pi^{-}$mass hypothesis is chosen to ensure all charmless two-body $b$-hadron decays are selected using the same criteria.

The events passing the trigger requirements are then filtered to further reduce the size of the data sample. In addition to tighter requirements on the kinematic variables already used in the software trigger, requirements on the larger of the transverse momenta $\left(p_{\mathrm{T}}^{h}\right)$ and of the impact parameters $\left(d_{\mathrm{IP}}^{h}\right)$ of the daughter particles are applied. As the rates of the various signals under study span two orders of magnitude, for efficient discrimination against combinatorial background three different sets of kinematic requirements are used to select events for: (A) the measurements of $\mathcal{B}\left(B^{0} \rightarrow \pi^{+} \pi^{-}\right) / \mathcal{B}\left(B^{0} \rightarrow K^{+} \pi^{-}\right)$, 


\begin{tabular}{|c|c|c|c|}
\hline Variable & Selection A & Selection B & Selection $\mathrm{C}$ \\
\hline Track $p_{\mathrm{T}}[\mathrm{GeV} / c]$ & $>1.1$ & $>1.2$ & $>1.2$ \\
$\operatorname{Track} d_{\mathrm{IP}}[\mu \mathrm{m}]$ & $>150$ & $>200$ & $>200$ \\
$\operatorname{Track} \chi^{2} / \mathrm{ndf}$ & $<3$ & $<3$ & $<3$ \\
$\max \left(p_{\mathrm{T}}^{h^{+}}, p_{\mathrm{T}}^{h^{\prime-}}\right)[\mathrm{GeV} / c]$ & $>2.8$ & $>3.0$ & $>3.0$ \\
$\max \left(d_{\mathrm{IP}}^{h^{+}}, d_{\mathrm{IP}}^{h^{\prime}}\right)[\mu \mathrm{m}]$ & $>300$ & $>400$ & $>400$ \\
$d_{\mathrm{CA}}[\mu \mathrm{m}]$ & $<80$ & $<80$ & $<80$ \\
$d_{\mathrm{IP}}^{B}[\mu \mathrm{m}]$ & $<60$ & $<60$ & $<60$ \\
$p_{\mathrm{T}}^{B}[\mathrm{GeV} / c]$ & $>2.2$ & $>2.4$ & $>2.8$ \\
$t_{\pi \pi}[\mathrm{ps}]$ & $>0.9$ & $>1.5$ & $>2.0$ \\
\hline
\end{tabular}

Table 1. Summary of criteria adopted in the event selections A, B and C defined in the text.

$\mathcal{B}\left(B_{s}^{0} \rightarrow K^{+} K^{-}\right) / \mathcal{B}\left(B^{0} \rightarrow K^{+} \pi^{-}\right)$and $\mathcal{B}\left(\Lambda_{b}^{0} \rightarrow p K^{-}\right) / \mathcal{B}\left(\Lambda_{b}^{0} \rightarrow p \pi^{-}\right) ; \quad$ (B) the measurement of $\mathcal{B}\left(B_{s}^{0} \rightarrow \pi^{+} K^{-}\right) / \mathcal{B}\left(B^{0} \rightarrow K^{+} \pi^{-}\right) ; \quad(\mathrm{C})$ the measurements of $\mathcal{B}\left(B^{0} \rightarrow K^{+} K^{-}\right) / \mathcal{B}\left(B_{s}^{0} \rightarrow K^{+} K^{-}\right) \quad$ and $\quad \mathcal{B}\left(B_{s}^{0} \rightarrow \pi^{+} \pi^{-}\right) / \mathcal{B}\left(B^{0} \rightarrow \pi^{+} \pi^{-}\right) . \quad$ The kinematic requirements adopted in each selection are summarized in table 1.

In order to evaluate the ratios of reconstruction efficiencies $\varepsilon_{\text {rec }}$, needed to calculate the relative branching fractions of two $H_{b} \rightarrow h^{+} h^{-}$decays, we apply selection and trigger requirements to fully simulated events. The results of this study are summarized in table 2 , where the uncertainties are due to the finite size of the simulated event samples. Other sources of systematic uncertainties are negligible at the current level of precision. This is confirmed by studies on samples of $D^{0}$ mesons decaying into pairs of charged hadrons, where reconstruction efficiencies are determined from data using measured signal yields and current world averages of the corresponding branching fractions. For the simulation, $p p$ collisions are generated using PYтніA 6.4 [15] with a specific LHCb configuration [16]. Decays of hadrons are described by EvTGEN [17] in which final state radiation is generated using Рнотоs [18]. The interaction of the generated particles with the detector and its response are implemented using the GEANT4 toolkit [19, 20] as described in ref. [21].

\section{Particle identification}

In order to disentangle the various $H_{b} \rightarrow h^{+} h^{\prime-}$ decay modes, the selected $b$-hadron candidates are divided into different final states using the PID capabilities of the two RICH detectors. Different sets of PID criteria are applied to the candidates passing the three selections, with PID discrimination power increasing from selection A to selection C. These criteria identify mutually exclusive sets of candidates. As discriminators we employ the quantities $\Delta \ln \mathcal{L}_{K \pi}$ and $\Delta \ln \mathcal{L}_{p \pi}$, or their difference $\Delta \ln \mathcal{L}_{K p}$ when appropriate, where $\Delta \ln \mathcal{L}_{\alpha \beta}$ is the difference between the natural logarithms of the likelihoods for a given daughter particle under mass hypotheses $\alpha$ and $\beta$, respectively. In order to determine the 


\begin{tabular}{|c|c|c|}
\hline Selection & Efficiency ratio & Value \\
\hline \multirow{2}{*}{$\mathrm{A}$} & $\varepsilon_{\mathrm{rec}}\left(B^{0} \rightarrow K^{+} \pi^{-}\right) / \varepsilon_{\mathrm{rec}}\left(B^{0} \rightarrow \pi^{+} \pi^{-}\right)$ & $0.98 \pm 0.02$ \\
& $\varepsilon_{\mathrm{rec}}\left(B^{0} \rightarrow K^{+} \pi^{-}\right) / \varepsilon_{\mathrm{rec}}\left(B_{s}^{0} \rightarrow K^{+} K^{-}\right)$ & $1.00 \pm 0.02$ \\
& $\varepsilon_{\mathrm{rec}}\left(\Lambda_{b}^{0} \rightarrow p K^{-}\right) / \varepsilon_{\mathrm{rec}}\left(\Lambda_{b}^{0} \rightarrow p \pi^{-}\right)$ & $1.00 \pm 0.02$ \\
\hline $\mathrm{B}$ & $\varepsilon_{\mathrm{rec}}\left(B^{0} \rightarrow K^{+} \pi^{-}\right) / \varepsilon_{\mathrm{rec}}\left(B_{s}^{0} \rightarrow \pi^{+} K^{-}\right)$ & $0.98 \pm 0.02$ \\
\hline \multirow{2}{*}{$\mathrm{C}$} & $\varepsilon_{\mathrm{rec}}\left(B^{0} \rightarrow \pi^{+} \pi^{-}\right) / \varepsilon_{\mathrm{rec}}\left(B_{s}^{0} \rightarrow \pi^{+} \pi^{-}\right)$ & $1.10 \pm 0.03$ \\
& $\varepsilon_{\mathrm{rec}}\left(B_{s}^{0} \rightarrow K^{+} K^{-}\right) / \varepsilon_{\mathrm{rec}}\left(B^{0} \rightarrow K^{+} K^{-}\right)$ & $0.92 \pm 0.02$ \\
\hline
\end{tabular}

Table 2. Ratios of reconstruction efficiencies of the various channels, as determined from Monte Carlo simulation, corresponding to the three event selections of table 1. PID efficiencies are not included here. The tight requirement on $t_{\pi \pi}$ used in selection $\mathrm{C}$ leads to a sizable difference from unity of the ratios in the last two rows, as the $B_{s}^{0} \rightarrow \pi^{+} \pi^{-}$and $B_{s}^{0} \rightarrow K^{+} K^{-}$decays proceed mainly via the short lifetime component of the $B_{s}^{0}$ meson.

corresponding PID efficiency for each two-body final state, a data-driven method is employed that uses $D^{*+} \rightarrow D^{0}\left(K^{-} \pi^{+}\right) \pi^{+}$and $\Lambda \rightarrow p \pi^{-}$decays as control samples. In this analysis about 6.7 million $D^{*+}$ decays and 4.2 million $\Lambda$ decays are used.

The production and decay kinematics of the $D^{0} \rightarrow K^{-} \pi^{+}$and $\Lambda \rightarrow p \pi^{-}$channels differ from those of the $b$-hadron decays under study. Since the RICH PID information is momentum dependent, a calibration procedure is performed by reweighting the $\Delta \ln \mathcal{L}_{\alpha \beta}$ distributions of true pions, kaons and protons obtained from the calibration samples, with the momentum distributions of daughter particles resulting from $H_{b} \rightarrow h^{+} h^{\prime-}$ decays. The $\Delta \ln \mathcal{L}_{\alpha \beta}$ and momentum distributions of the calibration samples and the momentum distributions of $H_{b}$ daughter particles are determined from data. In order to obtain background-subtracted distributions, extensive use of the sPlot technique [22] is made. This technique requires that extended maximum likelihood fits are performed, where signal and background components are modelled. It is achieved by fitting suitable models to the distribution of the variable $\delta m=m_{K \pi \pi}-m_{K \pi}$ for $D^{*+} \rightarrow D^{0}\left(K^{-} \pi^{+}\right) \pi^{+}$decays, to the $p \pi^{-}$mass for $\Lambda \rightarrow p \pi^{-}$decays and, for each of the three selections, to the invariant mass assuming the $\pi^{+} \pi^{-}$hypothesis for $H_{b} \rightarrow h^{+} h^{\prime-}$ decays. The variables $m_{K \pi \pi}$ and $m_{K \pi}$ are the reconstructed $D^{*+}$ and $D^{0}$ candidate masses, respectively.

In figure 1 the distributions of the variable $\delta m$ and of the invariant mass of $\Lambda \rightarrow p \pi^{-}$ are shown. The superimposed curves are the results of the maximum likelihood fits to the spectra.

The $D^{*+} \rightarrow D^{0}\left(K^{-} \pi^{+}\right) \pi^{+}$signal $\delta m$ spectrum has been modelled using the sum of three Gaussian functions $\left(G_{3}\right)$ with a common mean $(\mu)$, convolved with an empirical function which describes the asymmetric tail on the right-hand side of the spectrum:

$$
g(\delta m)=A\left[\Theta\left(\delta m^{\prime}-\mu\right) \cdot\left(\delta m^{\prime}-\mu\right)^{s}\right] \otimes G_{3}\left(\delta m-\delta m^{\prime}\right),
$$

where $A$ is a normalization factor, $\Theta$ is the Heaviside (step) function, $s$ is a free parameter determining the asymmetric shape of the distribution, $\otimes$ stands for convolution and the 

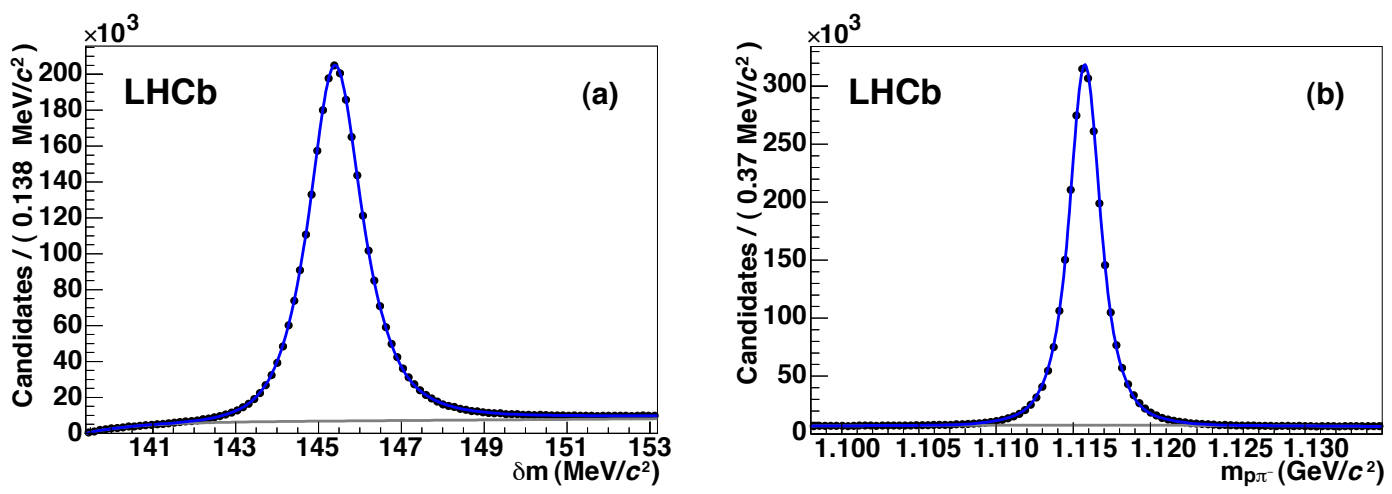

Figure 1. Distributions of (a) $\delta m=m_{K \pi \pi}-m_{K \pi}$ for $D^{*+} \rightarrow D^{0}\left(K^{-} \pi^{+}\right) \pi^{+}$candidates and (b) invariant mass of $\Lambda \rightarrow p \pi^{-}$candidates, used for the PID calibration. The curves are the results of maximum likelihood fits.

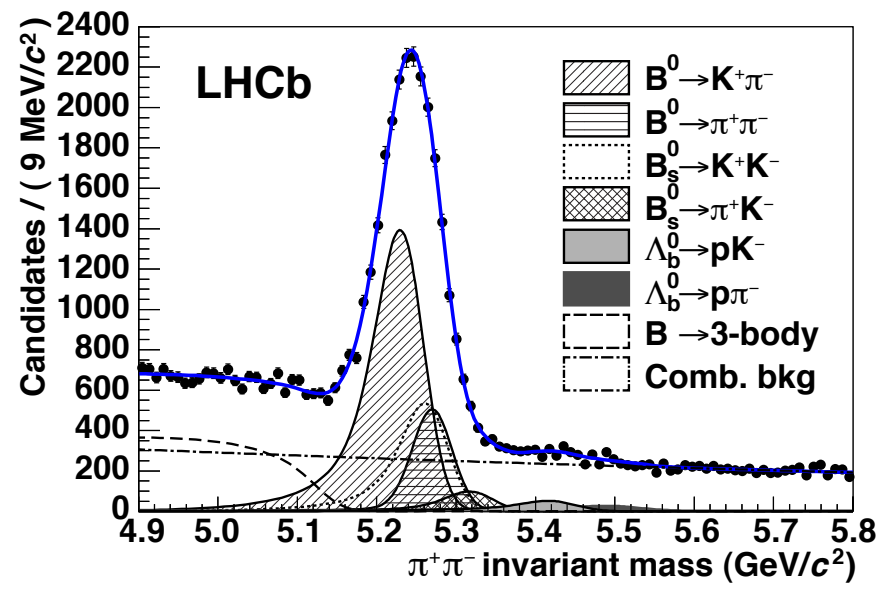

Figure 2. Invariant $\pi^{+} \pi^{-}$mass for candidates passing the selection A of table 1 . The result of an unbinned maximum likelihood fit is overlaid. The main contributions to the fit model are also shown.

convolution integral runs over $\delta m^{\prime}$. In order to model the background shape we use

$$
h(\delta m)=B\left[1-\exp \left(-\frac{\delta m-\delta m_{0}}{c}\right)\right],
$$

where $B$ is a normalization factor, and the free parameters $\delta m_{0}$ and $c$ govern the shape of the distribution. The fit to the $\Lambda \rightarrow p \pi^{-}$spectrum is made using a sum of three Gaussian functions for the signal and a second order polynomial for the background.

Figure 2 shows the invariant mass assuming the $\pi^{+} \pi^{-}$hypothesis for selected $b$-hadron candidates, using the kinematic selection $\mathrm{A}$ of table 1 and without applying any PID requirement. The shapes describing the various signal decay modes have been fixed by parameterizing the mass distributions obtained from Monte Carlo simulation convolved with a Gaussian resolution function with variable mean and width. The three-body and combinatorial backgrounds are modelled using an ARGUS function [23], convolved with the same Gaussian resolution function used for the signal distributions, and an exponential 

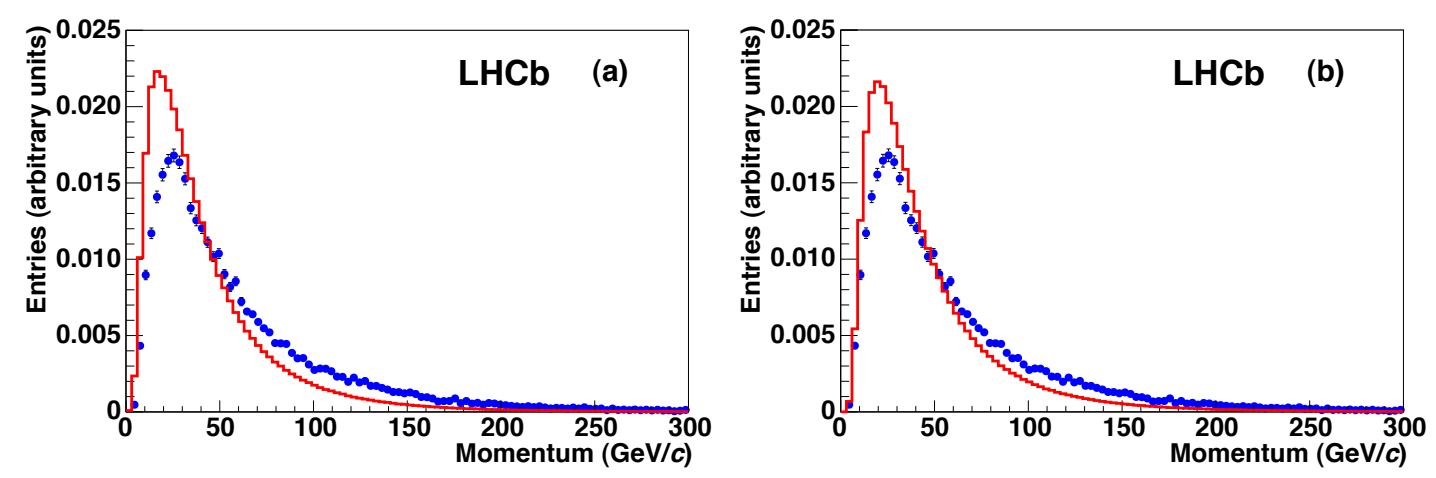

Figure 3. Momentum distributions of (a) pions and (b) kaons from $D^{0}$ decays in the PID calibration sample (histograms). For comparison, the points represent the inclusive momentum distribution of daughter particles in $H_{b} \rightarrow h^{+} h^{\prime-}$ decays. The distributions are normalized to the same area. This example corresponds to selection A.

function, respectively. The relative yields between the signal components have been fixed according to the known values of branching fractions and hadronization probabilities of $B^{0}, B_{s}^{0}$ and $\Lambda_{b}^{0}$ hadrons [24]. The fits corresponding to the kinematic selection criteria $\mathrm{B}$ and $\mathrm{C}$ of table 1 have also been made, although not shown, in order to take into account possible differences in the momentum distributions due to different selection criteria.

As mentioned above, the $s$ Plot procedure is used to determine the various $\Delta \ln \mathcal{L}_{\alpha \beta}$ and momentum distributions, and these are used to reweight the $D^{*+}$ and $\Lambda$ calibration samples. As an example, the momentum distributions of pions and kaons from $D^{0}$ decays and the inclusive momentum distribution of daughter particles in $H_{b} \rightarrow h^{+} h^{\prime-}$ decays, the latter corresponding to selection A, are shown in figure 3 .

The PID efficiencies corresponding to the three selections are determined by applying the PID selection criteria to the reweighted $D^{*+}$ and $\Lambda$ calibration samples. The results are reported in table 3. Using these efficiencies, the relevant PID efficiency ratios are determined and summarized in table 4. These ratios correspond to selection A only, since for the measurements involved in $\mathrm{B}$ and $\mathrm{C}$ the final states are identical and the ratios of PID efficiencies are equal to unity. It has been verified that the PID efficiencies do not show any sizeable dependence on the flavour of the parent hadron, as differences in the momentum distributions of the daughter particles for different parent hadrons are found to be small. Owing to the large sizes of the calibration samples, the uncertainties associated to the PID efficiency ratios are dominated by systematic effects, intrinsically related to the calibration procedure. They are estimated by means of a data-driven approach, where several fits to the $B^{0} \rightarrow K^{+} \pi^{-}$mass spectrum are made. The mass distributions in each fit are obtained by varying the PID selection criteria over a wide range, and then comparing the variation of the $B^{0} \rightarrow K^{+} \pi^{-}$signal yields determined by the fits to that of the PID efficiencies predicted by the calibration procedure. The largest deviation is then used to estimate the size of the systematic uncertainty. 


\begin{tabular}{|c|c|c|c|c|c|}
\hline Selection A & $\pi^{+} \pi^{-}$ & $K^{+} K^{-}$ & $K^{+} \pi^{-}$ & $p \pi^{-}$ & $p K^{-}$ \\
\hline$B^{0} \rightarrow \pi^{+} \pi^{-}$ & 43.1 & 0.33 & 28.6 & 1.53 & 0.13 \\
$B_{s}^{0} \rightarrow K^{+} K^{-}$ & 0.05 & 55.0 & 15.4 & 0.05 & 1.63 \\
$B_{(s)}^{0} \rightarrow K^{+} \pi^{-}$ & 1.40 & 4.17 & 67.9 & 0.72 & 0.06 \\
$\bar{B}_{(s)}^{0} \rightarrow \pi^{+} K^{-}$ & 1.40 & 4.17 & 2.09 & 0.02 & 0.85 \\
$\Lambda_{b}^{0} \rightarrow p \pi^{-}$ & 1.93 & 0.92 & 16.8 & 35.4 & 3.16 \\
$\bar{\Lambda}_{b}^{0} \rightarrow \pi^{+} \bar{p}$ & 1.93 & 0.92 & 0.95 & 0.03 & 0.18 \\
$\Lambda_{b}^{0} \rightarrow p K^{-}$ & 0.06 & 12.2 & 1.92 & 1.18 & 40.2 \\
$\bar{\Lambda}_{b}^{0} \rightarrow K^{+} \bar{p}$ & 0.06 & 12.2 & 4.51 & 0.03 & 0.18 \\
\hline
\end{tabular}

\begin{tabular}{|c|c|c|c|c|c|}
\hline Selection B & $\pi^{+} \pi^{-}$ & $K^{+} K^{-}$ & $K^{+} \pi^{-}$ & $p \pi^{-}$ & $p K^{-}$ \\
\hline$B^{0} \rightarrow \pi^{+} \pi^{-}$ & 42.8 & 0.33 & 2.06 & 1.51 & 0.13 \\
$B_{s}^{0} \rightarrow K^{+} K^{-}$ & 0.05 & 54.5 & 1.09 & 0.05 & 1.63 \\
$B_{(s)}^{0} \rightarrow K^{+} \pi^{-}$ & 1.38 & 4.12 & 35.7 & 0.72 & 0.06 \\
$\bar{B}_{(s)}^{0} \rightarrow \pi^{+} K^{-}$ & 1.38 & 4.12 & 0.02 & 0.02 & 0.84 \\
$\Lambda_{b}^{0} \rightarrow p \pi^{-}$ & 1.90 & 0.90 & 6.01 & 35.4 & 3.16 \\
$\bar{\Lambda}_{b}^{0} \rightarrow \pi^{+} \bar{p}$ & 1.90 & 0.90 & 0.03 & 0.03 & 0.17 \\
$\Lambda_{b}^{0} \rightarrow p K^{-}$ & 0.06 & 11.8 & 0.09 & 1.19 & 40.2 \\
$\bar{\Lambda}_{b}^{0} \rightarrow K^{+} \bar{p}$ & 0.06 & 11.8 & 0.88 & 0.03 & 0.17 \\
\hline
\end{tabular}

\begin{tabular}{|c|c|c|c|c|c|}
\hline Selection C & $\pi^{+} \pi^{-}$ & $K^{+} K^{-}$ & $K^{+} \pi^{-}$ & $p \pi^{-}$ & $p K^{-}$ \\
\hline$B^{0} \rightarrow \pi^{+} \pi^{-}$ & 40.5 & 0.00 & 1.64 & 1.51 & 0.00 \\
$B_{s}^{0} \rightarrow K^{+} K^{-}$ & 0.04 & 21.4 & 0.98 & 0.04 & 1.01 \\
$B_{(s)}^{0} \rightarrow K^{+} \pi^{-}$ & 1.27 & 0.11 & 32.4 & 0.70 & 0.00 \\
$\bar{B}_{(s)}^{0} \rightarrow \pi^{+} K^{-}$ & 1.27 & 0.11 & 0.01 & 0.02 & 0.54 \\
$\Lambda_{b}^{0} \rightarrow p \pi^{-}$ & 1.26 & 0.00 & 3.16 & 33.5 & 0.13 \\
$\bar{\Lambda}_{b}^{0} \rightarrow \pi^{+} \bar{p}$ & 1.26 & 0.00 & 0.02 & 0.02 & 0.03 \\
$\Lambda_{b}^{0} \rightarrow p K^{-}$ & 0.04 & 1.35 & 0.05 & 1.08 & 23.9 \\
$\bar{\Lambda}_{b}^{0} \rightarrow K^{+} \bar{p}$ & 0.04 & 1.35 & 0.65 & 0.02 & 0.03 \\
\hline
\end{tabular}

Table 3. PID efficiencies (in \%), for the various mass hypotheses, corresponding to the event samples passing the selections A, B and C of table 1. Different sets of PID requirements are applied in the three cases. 


\begin{tabular}{|c|c|}
\hline Efficiency ratio & Value \\
\hline$\varepsilon_{\mathrm{PID}}\left(K^{+} \pi^{-}\right) / \varepsilon_{\mathrm{PID}}\left(\pi^{+} \pi^{-}\right)$ & $1.57 \pm 0.09$ \\
$\varepsilon_{\mathrm{PID}}\left(K^{+} \pi^{-}\right) / \varepsilon_{\mathrm{PID}}\left(K^{+} K^{-}\right)$ & $1.23 \pm 0.06$ \\
$\varepsilon_{\mathrm{PID}}\left(p K^{-}\right) / \varepsilon_{\mathrm{PID}}\left(p \pi^{-}\right)$ & $1.14 \pm 0.05$ \\
\hline
\end{tabular}

Table 4. Ratios of PID efficiencies used to compute the relevant ratios of branching fractions, corresponding to selection A.
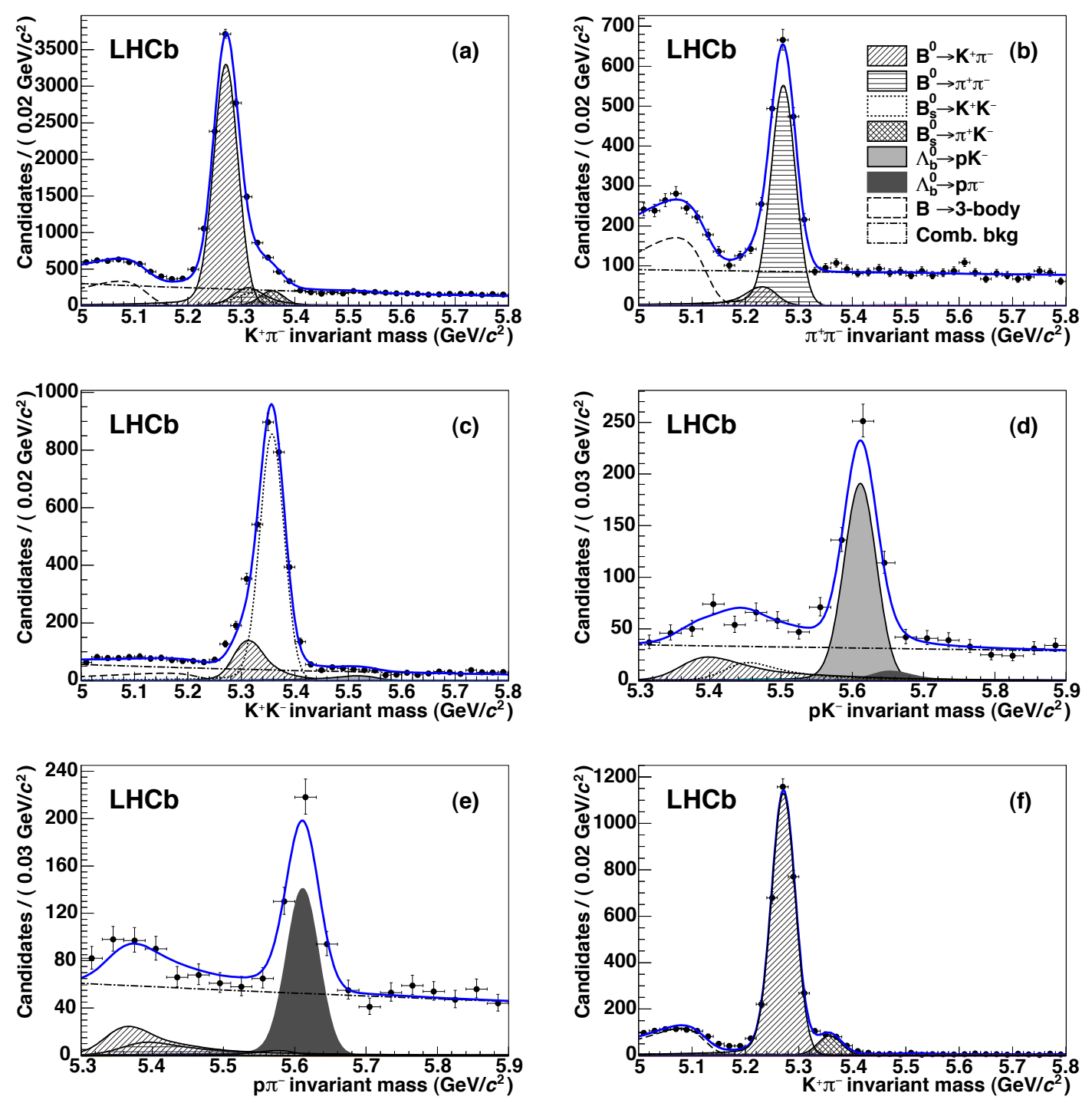

Figure 4. Invariant mass spectra corresponding to selection A for the mass hypotheses (a) $K^{+} \pi^{-}$, (b) $\pi^{+} \pi^{-}$, (c) $K^{+} K^{-}$, (d) $p K^{-}$and (e) $p \pi^{-}$, and to selection B for the mass hypothesis (f) $K^{+} \pi^{-}$. The results of the unbinned maximum likelihood fits are overlaid. The main components contributing to the fit model are also shown. 

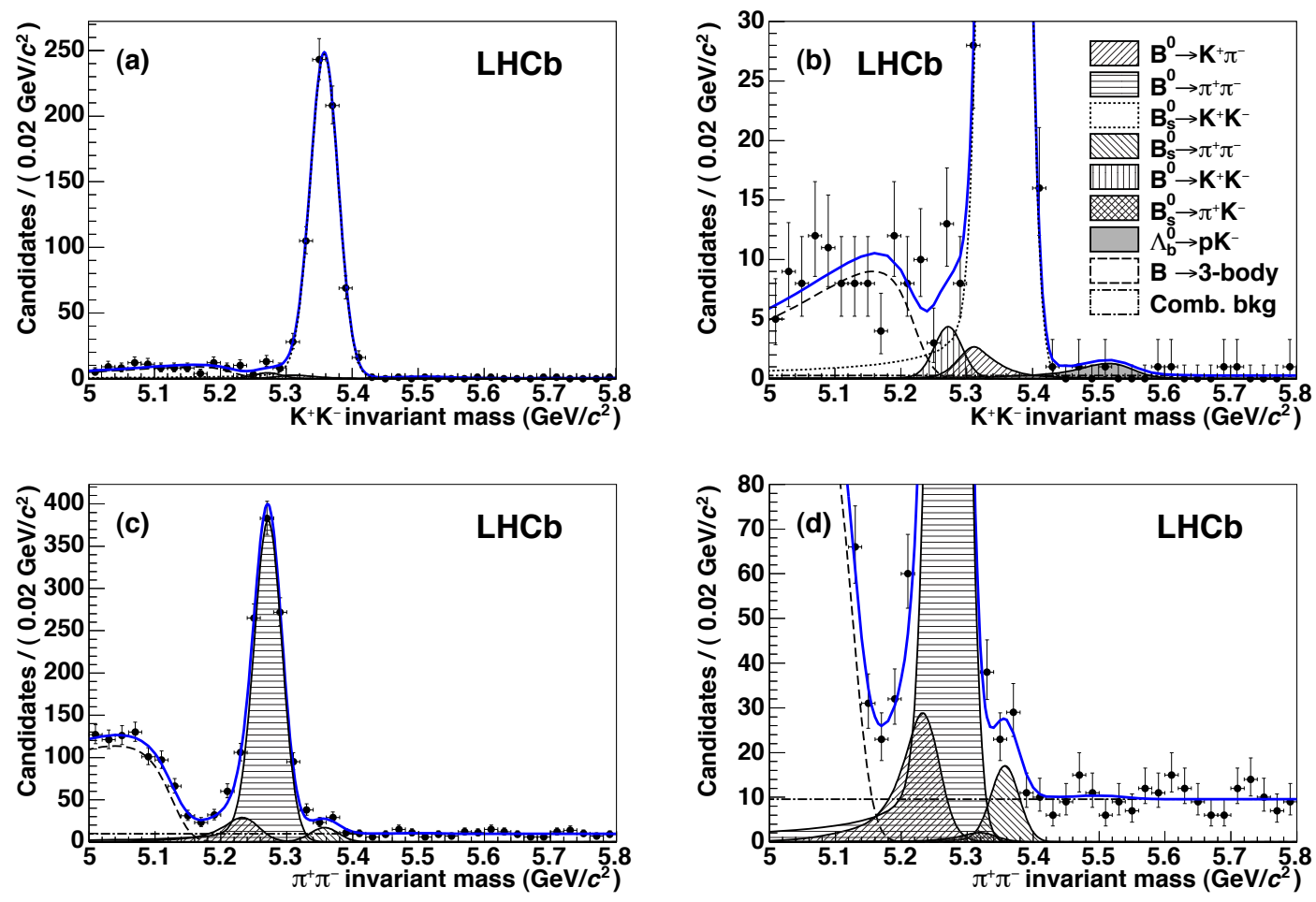

Figure 5. Invariant mass spectra corresponding to selection $\mathrm{C}$ for the mass hypotheses $(\mathrm{a}, \mathrm{b})$ $K^{+} K^{-}$and (c, d) $\pi^{+} \pi^{-}$. Plots (b) and (d) are the same as (a) and (c) respectively, but magnified to focus on the rare $B^{0} \rightarrow K^{+} K^{-}$and $B_{s}^{0} \rightarrow \pi^{+} \pi^{-}$signals. The results of the unbinned maximum likelihood fits are overlaid. The main components contributing to the fit model are also shown.

\section{Invariant mass fits to $H_{b} \rightarrow h^{+} h^{\prime-}$ spectra}

Unbinned maximum likelihood fits are performed to the mass spectra of events passing the selections A, B and C with associated PID selection criteria. For each selection we have five different spectra, corresponding to the final state hypotheses $K^{+} \pi^{-}, \pi^{+} \pi^{-}, K^{+} K^{-}$, $p K^{-}$and $p \pi^{-}$, to which we perform a simultaneous fit. Since each signal channel is also a background for all the other signal decay modes in case of misidentification of the final state particles (cross-feed background), the simultaneous fits to all the spectra allow a determination of the yields of the signal components together with those of the cross-feed backgrounds, once the appropriate PID efficiency factors are taken into account. The signal component for each hypothesis is described by a single Gaussian distribution, convolved with a function which describes the effect of the final state radiation on the mass line shape [25]. The combinatorial background is modelled by an exponential function and the shapes of the cross-feed backgrounds are obtained from Monte Carlo simulation. The background due to partially reconstructed three-body $B$ decays is parameterized by an ARGUS function [23] convolved with a Gaussian resolution function that has the same width as the signal distribution.

The overall mass resolution determined from the fits is about $22 \mathrm{MeV} / c^{2}$. Figure 4 shows the $K^{+} \pi^{-}, \pi^{+} \pi^{-}, K^{+} K^{-}, p K^{-}$and $p \pi^{-}$invariant mass spectra corresponding to selection A and the $K^{+} \pi^{-}$spectrum corresponding to selection B. Figure 5 shows the $\pi^{+} \pi^{-}$ 


\begin{tabular}{|c|c|c|}
\hline Selection & Decay & Signal yield \\
\hline & $B^{0} \rightarrow K^{+} \pi^{-}$ & $9822 \pm 122$ \\
& $B^{0} \rightarrow \pi^{+} \pi^{-}$ & $1667 \pm 51$ \\
$\mathrm{~A}$ & $B_{s}^{0} \rightarrow K^{+} K^{-}$ & $2523 \pm 59$ \\
& $\Lambda_{b}^{0} \rightarrow p K^{-}$ & $372 \pm 22$ \\
& $\Lambda_{b}^{0} \rightarrow p \pi^{-}$ & $279 \pm 22$ \\
\hline $\mathrm{B}$ & $B^{0} \rightarrow K^{+} \pi^{-}$ & $3295 \pm 59$ \\
& $B_{s}^{0} \rightarrow \pi^{+} K^{-}$ & $249 \pm 20$ \\
\hline & $B^{0} \rightarrow \pi^{+} \pi^{-}$ & $1076 \pm 36$ \\
$\mathrm{C}$ & $B_{s}^{0} \rightarrow K^{+} K^{-}$ & $682 \pm 27$ \\
& $B^{0} \rightarrow K^{+} K^{-}$ & $13_{-5}^{+6}$ \\
& $B_{s}^{0} \rightarrow \pi^{+} \pi^{-}$ & $49_{-9}^{+11}$ \\
\hline
\end{tabular}

Table 5. Signal yields determined by the unbinned maximum likelihood fits to the data samples surviving the event selections A, B and C of table 1 with the associated PID criteria. Only statistical uncertainties are shown.

\begin{tabular}{|c|c|c|}
\hline Selection & Ratio & Value \\
\hline \multirow{2}{*}{$\mathrm{A}$} & $\frac{N\left(B^{0} \rightarrow \pi^{+} \pi^{-}\right)}{N\left(B^{0} \rightarrow K^{+} \pi^{-}\right)}$ & $0.170 \pm 0.006$ \\
& $\frac{N\left(B_{s}^{0} \rightarrow K^{+} K^{-}\right)}{N\left(B^{0} \rightarrow K^{+} \pi^{-}\right)}$ & $0.257 \pm 0.007$ \\
& $\frac{N\left(\Lambda_{b}^{0} \rightarrow p \pi^{-}\right)}{N\left(\Lambda_{b}^{0} \rightarrow p K^{-}\right)}$ & $0.75 \pm 0.07$ \\
\hline $\mathrm{B}$ & $\frac{N\left(B_{s}^{0} \rightarrow \pi^{+} K^{-}\right)}{N\left(B^{0} \rightarrow K^{+} \pi^{-}\right)}$ & $0.076 \pm 0.006$ \\
\hline $\mathrm{C}$ & $\frac{N\left(B^{0} \rightarrow K^{+} K^{-}\right)}{N\left(B_{s}^{0} \rightarrow K^{+} K^{-}\right)}$ & $0.019_{-0.007}^{+0.009}$ \\
& $\frac{N\left(B_{s}^{0} \rightarrow \pi^{+} \pi^{-}\right)}{N\left(B^{0} \rightarrow \pi^{+} \pi^{-}\right)}$ & $0.046_{-0.009}^{+0.010}$ \\
\hline
\end{tabular}

Table 6. Ratios of signal yields needed for the measurement of the relative branching fractions. Only statistical uncertainties are shown.

and $K^{+} K^{-}$mass spectra corresponding to selection C. As is apparent in the latter, while a $B_{s}^{0} \rightarrow \pi^{+} \pi^{-}$mass peak is visible above the combinatorial background, there are not yet sufficient data to observe a significant $B^{0} \rightarrow K^{+} K^{-}$signal. As an additional complication, the mass peak of the $B^{0} \rightarrow K^{+} K^{-}$decay is expected in a region where various components give non-negligible contributions, in particular the radiative tail of the $B_{s}^{0} \rightarrow K^{+} K^{-}$decay and the $B^{0} \rightarrow K^{+} \pi^{-}$cross-feed background. The relevant event yields for each of the three selections are summarized in table 5 . Using the values listed in table 5 , we can calculate the ratios of yields needed to compute the relative branching fractions. These ratios are given in table 6 , with their statistical uncertainties. 


\begin{tabular}{|c|c|c|c|c|c|c|}
\hline Syst. uncertainty & $\frac{N\left(B^{0} \rightarrow \pi^{+} \pi^{-}\right)}{N\left(B^{0} \rightarrow K^{+} \pi^{-}\right)}$ & $\frac{N\left(B_{s}^{0} \rightarrow K^{+} K^{-}\right)}{N\left(B^{0} \rightarrow K^{+} \pi^{-}\right)}$ & $\frac{N\left(\Lambda_{b}^{0} \rightarrow p \pi^{-}\right)}{N\left(\Lambda_{b}^{0} \rightarrow p K^{-}\right)}$ & $\frac{N\left(B_{s}^{0} \rightarrow \pi^{+} K^{-}\right)}{N\left(B^{0} \rightarrow K^{+} \pi^{-}\right)}$ & $\frac{N\left(B^{0} \rightarrow K^{+} K^{-}\right)}{N\left(B_{s}^{0} \rightarrow K^{+} K^{-}\right)}$ & $\frac{N\left(B_{s}^{0} \rightarrow \pi^{+} \pi^{-}\right)}{N\left(B^{0} \rightarrow \pi^{+} \pi^{-}\right)}$ \\
\hline PID calibration & 0.0002 & 0.0012 & 0.0075 & 0.0013 & 0.0005 & 0.0002 \\
Final state rad. & 0.0019 & 0.0043 & 0.0140 & 0.0012 & 0.0093 & 0.0013 \\
Signal model & negligible & 0.0001 & 0.0013 & 0.0052 & 0.0010 & 0.0031 \\
Comb. bkg model & 0.0013 & 0.0006 & 0.0086 & negligible & 0.0012 & 0.0004 \\
$K \pi$ 3-body bkg & 0.0018 & 0.0048 & 0.0239 & 0.0011 & negligible & negligible \\
Cross-feed bkg & 0.0023 & 0.0045 & 0.0042 & 0.0008 & 0.0008 & 0.0002 \\
\hline Total & 0.0038 & 0.0080 & 0.0304 & 0.0056 & 0.0095 & 0.0034 \\
\hline
\end{tabular}

Table 7. Systematic uncertainties on the ratios of signal yields. The total systematic uncertainties are obtained by summing the individual contributions in quadrature.

\section{Systematic uncertainties}

The systematic uncertainties on the ratios of signal yields are related to the PID calibration and to the modelling of the signal and background components in the maximum likelihood fits. Knowledge of PID efficiencies is necessary to compute the number of cross-feed background events affecting the fit of any $H_{b}$ mass spectrum. In order to estimate the impact of imperfect PID calibration, we perform unbinned maximum likelihood fits after having altered the number of cross-feed background events present in the relevant mass spectra according to the systematic uncertainties affecting the PID efficiencies. An estimate of the uncertainty due to possible imperfections in the description of the final state radiation is determined by varying, over a wide range, the amount of emitted radiation [25] in the signal line shape parameterization. The possibility of an incorrect description of the core distribution in the signal mass model is investigated by replacing the single Gaussian with the sum of two Gaussian functions with a common mean. The impact of additional three-body $B$ decays in the $K^{+} \pi^{-}$spectrum, not accounted for in the baseline fit - namely $B \rightarrow \pi \pi \pi$ where one pion is missed in the reconstruction and another is misidentified as a kaon is investigated. The mass line shape of this background component is determined from Monte Carlo simulation, and the fit is repeated after having modified the baseline parameterization accordingly. For the modelling of the combinatorial background component, the fit is repeated using a first-order polynomial. Finally, for the cross-feed backgrounds, two distinct systematic uncertainties are estimated: one due to a relative bias in the mass scale of the simulated distributions with respect to the signal distributions in data, and another accounting for the difference in mass resolution between simulation and data. All the shifts from the relevant baseline values are accounted for as systematic uncertainties. A summary of all systematic uncertainties on the ratios of event yields is reported in table 7 . The total uncertainties are obtained by summing the individual contributions in quadrature. The uncertainties on the ratios of reconstruction and PID efficiencies, reported in tables 2 and 4, are also included in the computation of the total systematic uncertainties on the ratios of branching fractions, reported in the next section. 


\section{$6 \quad$ Results and conclusions}

The following quantities are determined using eq. (1.1) and the values reported in tables 2, 4, 6 and 7 :

$$
\begin{aligned}
\mathcal{B}\left(B^{0} \rightarrow \pi^{+} \pi^{-}\right) / \mathcal{B}\left(B^{0} \rightarrow K^{+} \pi^{-}\right) & =0.262 \pm 0.009 \pm 0.017 \\
\left(f_{s} / f_{d}\right) \cdot \mathcal{B}\left(B_{s}^{0} \rightarrow K^{+} K^{-}\right) / \mathcal{B}\left(B^{0} \rightarrow K^{+} \pi^{-}\right) & =0.316 \pm 0.009 \pm 0.019 \\
\left(f_{s} / f_{d}\right) \cdot \mathcal{B}\left(B_{s}^{0} \rightarrow \pi^{+} K^{-}\right) / \mathcal{B}\left(B^{0} \rightarrow K^{+} \pi^{-}\right) & =0.074 \pm 0.006 \pm 0.006 \\
\left(f_{d} / f_{s}\right) \cdot \mathcal{B}\left(B^{0} \rightarrow K^{+} K^{-}\right) / \mathcal{B}\left(B_{s}^{0} \rightarrow K^{+} K^{-}\right) & =0.018_{-0.007}^{+0.008} \pm 0.009 \\
\left(f_{s} / f_{d}\right) \cdot \mathcal{B}\left(B_{s}^{0} \rightarrow \pi^{+} \pi^{-}\right) / \mathcal{B}\left(B^{0} \rightarrow \pi^{+} \pi^{-}\right) & =0.050_{-0.009}^{+0.011} \pm 0.004 \\
\mathcal{B}\left(\Lambda_{b}^{0} \rightarrow p \pi^{-}\right) / \mathcal{B}\left(\Lambda_{b}^{0} \rightarrow p K^{-}\right) & =0.86 \pm 0.08 \pm 0.05
\end{aligned}
$$

where the first uncertainties are statistical and the second systematic. Using the current world average $\mathcal{B}\left(B^{0} \rightarrow K^{+} \pi^{-}\right)=(19.4 \pm 0.6) \times 10^{-6}$ provided by the Heavy Flavor Averaging Group [24], and our measurement of the ratio between the $b$-quark hadronization probabilities $f_{s} / f_{d}=0.267_{-0.020}^{+0.021}[26]$, we obtain the following branching fractions:

$$
\begin{aligned}
\mathcal{B}\left(B^{0} \rightarrow \pi^{+} \pi^{-}\right) & =(5.08 \pm 0.17 \pm 0.37) \times 10^{-6}, \\
\mathcal{B}\left(B_{s}^{0} \rightarrow K^{+} K^{-}\right) & =(23.0 \pm 0.7 \pm 2.3) \times 10^{-6}, \\
\mathcal{B}\left(B_{s}^{0} \rightarrow \pi^{+} K^{-}\right) & =(5.4 \pm 0.4 \pm 0.6) \times 10^{-6} \\
\mathcal{B}\left(B^{0} \rightarrow K^{+} K^{-}\right) & =\left(0.11_{-0.04}^{+0.05} \pm 0.06\right) \times 10^{-6} \\
\mathcal{B}\left(B_{s}^{0} \rightarrow \pi^{+} \pi^{-}\right) & =\left(0.95_{-0.17}^{+0.21} \pm 0.13\right) \times 10^{-6},
\end{aligned}
$$

where the systematic uncertainties include the uncertainties on $\mathcal{B}\left(B^{0} \rightarrow K^{+} \pi^{-}\right)$and $f_{s} / f_{d}$.

These results are compatible with the current experimental averages [24] and with available theoretical predictions [27-37]. The measurements of $\mathcal{B}\left(B_{s}^{0} \rightarrow K^{+} K^{-}\right)$, $\mathcal{B}\left(B_{s}^{0} \rightarrow \pi^{+} K^{-}\right), \mathcal{B}\left(B^{0} \rightarrow K^{+} K^{-}\right)$and $\mathcal{B}\left(\Lambda_{b}^{0} \rightarrow p \pi^{-}\right) / \mathcal{B}\left(\Lambda_{b}^{0} \rightarrow p K^{-}\right)$are the most precise to date. Using a likelihood ratio test and including the systematic uncertainties on the signal yield, we obtain for the $B_{s}^{0} \rightarrow \pi^{+} \pi^{-}$signal a significance of $5.3 \sigma$. This significance is estimated as $s_{\text {stat }}=\sqrt{-2 \ln \frac{\mathcal{L}_{\mathrm{B}}}{\mathcal{L}_{\mathrm{S}+\mathrm{B}}}}$, where $\mathcal{L}_{\mathrm{S}+\mathrm{B}}$ and $\mathcal{L}_{\mathrm{B}}$ are the values of the likelihoods at the maximum in the two cases of signal-plus-background and background-only hypotheses, respectively. The value of $s_{\text {stat }}=5.5 \sigma$ is then corrected by taking into account the systematic uncertainty as $s_{\text {tot }}=s_{\text {stat }} / \sqrt{1+\sigma_{\text {syst }}^{2} / \sigma_{\text {stat }}^{2}}$, where $\sigma_{\text {stat }}$ and $\sigma_{\text {syst }}$ are the statistical and systematic uncertainties. This is the first observation at more than $5 \sigma$ of the $B_{s}^{0} \rightarrow \pi^{+} \pi^{-}$decay.

\section{Acknowledgments}

We express our gratitude to our colleagues in the CERN accelerator departments for the excellent performance of the LHC. We thank the technical and administrative staff at CERN and at the LHCb institutes, and acknowledge support from the National Agencies: CAPES, CNPq, FAPERJ and FINEP (Brazil); CERN; NSFC (China); CNRS/IN2P3 (France); BMBF, DFG, HGF and MPG (Germany); SFI (Ireland); INFN (Italy); FOM 
and NWO (The Netherlands); SCSR (Poland); ANCS (Romania); MinES of Russia and Rosatom (Russia); MICINN, XuntaGal and GENCAT (Spain); SNSF and SER (Switzerland); NAS Ukraine (Ukraine); STFC (United Kingdom); NSF (USA). We also acknowledge the support received from the ERC under FP7 and the Region Auvergne.

Open Access. This article is distributed under the terms of the Creative Commons Attribution License which permits any use, distribution and reproduction in any medium, provided the original author(s) and source are credited.

\section{References}

[1] N. Cabibbo, Unitary symmetry and leptonic decays, Phys. Rev. Lett. 10 (1963) 531 [inSPIRE].

[2] M. Kobayashi and T. Maskawa, CP violation in the renormalizable theory of weak interaction, Prog. Theor. Phys. 49 (1973) 652 [InSPIRE].

[3] R. Fleischer, New strategies to extract $\beta$ and $\gamma$ from $B_{d} \rightarrow \pi^{+} \pi^{-}$and $B_{s} \rightarrow K^{+} K^{-}$, Phys. Lett. B 459 (1999) 306 [hep-ph/9903456] [INSPIRE].

[4] M. Gronau and J.L. Rosner, The role of $B_{s} \rightarrow K \pi$ in determining the weak phase $\gamma$, Phys. Lett. B 482 (2000) 71 [hep-ph/0003119] [INSPIRE].

[5] H.J. Lipkin, Is observed direct CP-violation in $B_{d} \rightarrow K^{+} \pi^{-}$due to new physics? Check standard model prediction of equal violation in $B_{s} \rightarrow K^{-} \pi^{+}$, Phys. Lett. B 621 (2005) 126 [hep-ph/0503022] [INSPIRE].

[6] R. Fleischer, $B_{s, d} \rightarrow \pi \pi, \pi K, K K$ : status and prospects, Eur. Phys. J. C 52 (2007) 267 [arXiv:0705.1121] [INSPIRE].

[7] R. Fleischer and R. Knegjens, In pursuit of new physics with $B_{s}^{0} \rightarrow K^{+} K^{-}$, Eur. Phys. J. C 71 (2011) 1532 [arXiv:1011.1096] [INSPIRE].

[8] BABAR collaboration, B. Aubert et al., Measurement of CP asymmetries and branching fractions in $B^{0} \rightarrow \rightarrow \pi^{+} \pi^{-}, B^{0} \rightarrow K^{+} \pi^{-}, B^{0} \rightarrow \pi^{0} \pi^{0}, B^{0} \rightarrow K^{0} \pi^{0}$ and isospin analysis of $B \rightarrow \pi \pi$ decays, arXiv:0807.4226 [INSPIRE].

[9] Belle collaboration, S. Lin et al., Difference in direct charge-parity violation between charged and neutral B meson decays, Nature 452 (2008) 332 [INSPIRE].

[10] CDF collaboration, T. Aaltonen et al., Measurements of direct CP-violating asymmetries in charmless decays of strange bottom mesons and bottom baryons, Phys. Rev. Lett. 106 (2011) 181802 [arXiv: 1103.5762] [INSPIRE].

[11] LHCB collaboration, R. Aaij et al., First evidence of direct CP-violation in charmless two-body decays of $B_{s}^{0}$ mesons, Phys. Rev. Lett. 108 (2012) 201601 [arXiv:1202.6251] [INSPIRE].

[12] CDF collaboration, T. Aaltonen et al., Evidence for the charmless annihilation decay mode $B_{s}^{0} \rightarrow \pi^{+} \pi^{-}$, Phys. Rev. Lett. 108 (2012) 211803 [arXiv:1111.0485] [INSPIRE].

[13] LHCB collaboration, J. Alves, A. Augusto et al., The LHCb detector at the LHC, 2008 JINST 3 S08005 [INSPIRE].

[14] V. Gligorov, C. Thomas and M. Williams, The HLT inclusive B triggers, LHCb-PUB-2011-016 (2011). 
[15] T. Sjöstrand, S. Mrenna and P.Z. Skands, PYTHIA 6.4 physics and manual, JHEP 05 (2006) 026 [hep-ph/0603175] [INSPIRE].

[16] I. Belyaev et al., Handling of the generation of primary events in Gauss, the LHCb simulation framework, Nucl. Sci. Symp. Conf. Rec. (2010) 1155.

[17] D. Lange, The EvtGen particle decay simulation package, Nucl. Instrum. Meth. A 462 (2001) 152 [INSPIRE].

[18] P. Golonka and Z. Was, PHOTOS Monte Carlo: a precision tool for QED corrections in Z and $W$ decays, Eur. Phys. J. C 45 (2006) 97 [hep-ph/0506026] [INSPIRE].

[19] GEANT4 collaboration, J. Allison et al., GEANT4 developments and applications, IEEE Trans. Nucl. Sci. 53 (2006) 270.

[20] GEANT4 collaboration, S. Agostinelli et al., GEANT4: a simulation toolkit, Nucl. Instrum. Meth. A 506 (2003) 250 [INSPIRE].

[21] M. Clemencic et al., The LHCb simulation application, Gauss: design, evolution and experience, J. Phys. Conf. Ser. 331 (2011) 032023.

[22] M. Pivk and F.R. Le Diberder, SPlot: a statistical tool to unfold data distributions, Nucl. Instrum. Meth. A 555 (2005) 356 [physics/0402083] [INSPIRE].

[23] ARGUS collaboration, H. Albrecht et al., Search for $b \rightarrow s \gamma$ in exclusive decays of $B$ mesons, Phys. Lett. B 229 (1989) 304 [INSPIRE].

[24] Heavy Flavor Averaging Group collaboration, D. Asner et al., Averages of b-hadron, c-hadron and $\tau$-lepton properties, arXiv:1010.1589, updates available online at http://www.slac.stanford.edu/xorg/hfag [INSPIRE].

[25] E. Baracchini and G. Isidori, Electromagnetic corrections to non-leptonic two-body $B$ and $D$ decays, Phys. Lett. B 633 (2006) 309 [hep-ph/0508071] [INSPIRE].

[26] LHCB collaboration, R. Aaij et al., Measurement of b hadron production fractions in $7 \mathrm{TeV}$ pp collisions, Phys. Rev. D 85 (2012) 032008 [arXiv:1111.2357] [InSPIRE].

[27] H.-n. Li, S. Mishima and A. Sanda, Resolution to the $B \rightarrow \pi K$ puzzle, Phys. Rev. D 72 (2005) 114005 [hep-ph/0508041] [INSPIRE].

[28] C.-D. Lu, Y.-L. Shen and W. Wang, Final state interaction in $B \rightarrow K K$ decays, Phys. Rev. D 73 (2006) 034005 [hep-ph/0511255] [INSPIRE].

[29] S. Descotes-Genon, J. Matias and J. Virto, Exploring $B_{d, s} \rightarrow K K$ decays through flavour symmetries and QCD-factorisation, Phys. Rev. Lett. 97 (2006) 061801 [hep-ph/0603239] [INSPIRE].

[30] H.-Y. Cheng and C.-K. Chua, Revisiting charmless hadronic $B_{u, d}$ decays in $Q C D$ factorization, Phys. Rev. D 80 (2009) 114008 [arXiv: 0909.5229] [inSPIRE].

[31] A.R. Williamson and J. Zupan, Two body B decays with isosinglet final states in SCET, Phys. Rev. D 74 (2006) 014003 [Erratum ibid. D 74 (2006) 03901] [hep-ph/0601214] [INSPIRE].

[32] A. Ali, G. Kramer, Y. Li, C.-D. Lu, Y.-L. Shen, et al., Charmless non-leptonic $B_{s}$ decays to $P P, P V$ and $V V$ final states in the $p Q C D$ approach, Phys. Rev. D 76 (2007) 074018 [hep-ph/0703162] [INSPIRE].

[33] J. Liu, R. Zhou and Z.-J. Xiao, $B_{s} \rightarrow P P$ decays and the NLO contributions in the $p Q C D$ Approach, arXiv:0812.2312 [INSPIRE]. 
[34] H.-Y. Cheng and C.-K. Chua, QCD Factorization for charmless hadronic $B_{s}$ decays revisited, Phys. Rev. D 80 (2009) 114026 [arXiv:0910.5237] [InSPIRE].

[35] R. Mohanta, A. Giri and M. Khanna, Charmless two-body hadronic decays of $\Lambda_{b}$ baryon, Phys. Rev. D 63 (2001) 074001 [hep-ph/0006109] [InSPIRE].

[36] H. Kaur and M. Khanna, Charmless weak decays of $\Lambda_{b}$ baryon, Int. J. Mod. Phys. A 21 (2006) 121 [INSPIRE].

[37] C.D. Lü, Y.M. Wang, H. Zou, A. Ali and G. Kramer, Anatomy of the $p Q C D$ approach to the baryonic decays $\Lambda_{b} \rightarrow p \pi, p K$, Phys. Rev. D 80 (2009) 034011 [arXiv:0906.1479] [INSPIRE]. 


\section{The LHCb collaboration}

R. Aaij ${ }^{38}$, C. Abellan Beteta ${ }^{33, n}$, B. Adeva ${ }^{34}$, M. Adinolfi ${ }^{43}$, C. Adrover ${ }^{6}$, A. Affolder ${ }^{49}$, Z. Ajaltouni ${ }^{5}$, J. Albrecht ${ }^{35}$, F. Alessio ${ }^{35}$, M. Alexander ${ }^{48}$, S. Ali ${ }^{38}$, G. Alkhazov ${ }^{27}$, P. Alvarez Cartelle ${ }^{34}$, A.A. Alves Jr22, S. Amato ${ }^{2}$, Y. Amhis ${ }^{36}$, J. Anderson ${ }^{37}$, R.B. Appleby ${ }^{51}$, O. Aquines Gutierrez ${ }^{10}$, F. Archilli ${ }^{18,35}$, A. Artamonov ${ }^{32}$, M. Artuso ${ }^{53,35}$, E. Aslanides ${ }^{6}$, G. Auriemma ${ }^{22, m}$, S. Bachmann ${ }^{11}$, J.J. Back ${ }^{45}$, V. Balagura ${ }^{28,35}$, W. Baldini ${ }^{16}$, R.J. Barlow ${ }^{51}$, C. Barschel ${ }^{35}$, S. Barsuk ${ }^{7}$, W. Barter ${ }^{44}$, A. Bates ${ }^{48}$, C. Bauer ${ }^{10}$, Th. Bauer ${ }^{38}$, A. Bay ${ }^{36}$, I. Bediaga ${ }^{1}$, S. Belogurov ${ }^{28}$, K. Belous ${ }^{32}$, I. Belyaev ${ }^{28}$, E. Ben-Haim ${ }^{8}$, M. Benayoun ${ }^{8}$, G. Bencivenni ${ }^{18}$, S. Benson ${ }^{47}$, J. Benton ${ }^{43}$, R. Bernet ${ }^{37}$, M.-O. Bettler ${ }^{17}$, M. van Beuzekom ${ }^{38}$, A. Bien ${ }^{11}$, S. Bifani ${ }^{12}$, T. $\operatorname{Bird}^{51}$, A. Bizzeti ${ }^{17, h}$, P.M. Bjørnstad ${ }^{51}$, T. Blake ${ }^{35}$, F. Blanc ${ }^{36}$, C. Blanks ${ }^{50}$, J. Blouw ${ }^{11}$, S. Blusk ${ }^{53}$, A. Bobrov ${ }^{31}$, V. Bocci ${ }^{22}$, A. Bondar ${ }^{31}$, N. Bondar ${ }^{27}$, W. Bonivento ${ }^{15}$, S. Borghi ${ }^{48,51}$, A. Borgia ${ }^{53}$, T.J.V. Bowcock ${ }^{49}$, C. Bozzi ${ }^{16}$, T. Brambach ${ }^{9}$, J. van den Brand ${ }^{39}$, J. Bressieux ${ }^{36}$, D. Brett ${ }^{51}$, M. Britsch ${ }^{10}$, T. Britton ${ }^{53}$, N.H. Brook ${ }^{43}$, H. Brown ${ }^{49}$, K. de Bruyn ${ }^{38}$, A. Büchler-Germann ${ }^{37}$, I. Burducea ${ }^{26}$, A. Bursche ${ }^{37}$, J. Buytaert ${ }^{35}$, S. Cadeddu ${ }^{15}$, O. Callot ${ }^{7}$, M. Calvi ${ }^{20, j}$, M. Calvo Gomez ${ }^{33, n}$, A. Camboni ${ }^{33}$, P. Campana ${ }^{18,35}$, A. Carbone ${ }^{14}$, G. Carboni ${ }^{21, k}$, R. Cardinale ${ }^{19, i, 35}$, A. Cardini ${ }^{15}$, L. Carson ${ }^{50}$, K. Carvalho Akiba ${ }^{2}$, G. Casse ${ }^{49}$, M. Cattaneo ${ }^{35}$, Ch. Cauet ${ }^{9}$, M. Charles ${ }^{52}$, Ph. Charpentier ${ }^{35}$, N. Chiapolini ${ }^{37}$, K. Ciba ${ }^{35}$, X. Cid Vidal ${ }^{34}$, G. Ciezarek $^{50}$, P.E.L. Clarke ${ }^{47,35}$, M. Clemencic ${ }^{35}$, H.V. Cliff ${ }^{44}$, J. Closier ${ }^{35}$, C. Coca ${ }^{26}$, V. Coco $^{38}$, J. Cogan ${ }^{6}$, P. Collins ${ }^{35}$, A. Comerma-Montells ${ }^{33}$, A. Contu ${ }^{52}$, A. Cook ${ }^{43}$, M. Coombes $^{43}$, G. Corti $^{35}$, B. Couturier ${ }^{35}$, G.A. Cowan $^{36}$, R. Currie ${ }^{47}$, C. D'Ambrosio ${ }^{35}$, P. David ${ }^{8}$, P.N.Y. David ${ }^{38}$, I. De Bonis ${ }^{4}$, S. De Capua ${ }^{21, k}$, M. De Cian ${ }^{37}$, J.M. De Miranda ${ }^{1}$, L. De Paula ${ }^{2}$, P. De Simone ${ }^{18}$, D. Decamp ${ }^{4}$, M. Deckenhoff ${ }^{9}$, H. Degaudenzi ${ }^{36,35}$, L. Del Buono ${ }^{8}$, C. Deplano ${ }^{15}$, D. Derkach ${ }^{14,35}$, O. Deschamps ${ }^{5}$, F. Dettori ${ }^{39}$, J. Dickens ${ }^{44}$, H. Dijkstra ${ }^{35}$, P. Diniz Batista ${ }^{1}$, F. Domingo Bonal ${ }^{33, n}$, S. Donleavy ${ }^{49}$, F. Dordei ${ }^{11}$, A. Dosil Suárez ${ }^{34}$, D. Dossett ${ }^{45}$, A. Dovbnya ${ }^{40}$, F. Dupertuis ${ }^{36}$, R. Dzhelyadin ${ }^{32}$, A. Dziurda $^{23}$, S. Easo ${ }^{46}$, U. Egede ${ }^{50}$, V. Egorychev ${ }^{28}$, S. Eidelman ${ }^{31}$, D. van Eijk ${ }^{38}$, F. Eisele ${ }^{11}$, S. Eisenhardt ${ }^{47}$, R. Ekelhof ${ }^{9}$, L. Eklund ${ }^{48}$, Ch. Elsasser $^{37}$, D. Elsby ${ }^{42}$, D. Esperante Pereira $^{34}$, A. Falabella ${ }^{16, e, 14}$, C. Färber ${ }^{11}$, G. Fardell ${ }^{47}$, C. Farinelli ${ }^{38}$, S. Farry ${ }^{12}$, V. Fave ${ }^{36}$, V. Fernandez Albor ${ }^{34}$, M. Ferro-Luzzi ${ }^{35}$, S. Filippov ${ }^{30}$, C. Fitzpatrick ${ }^{47}$, M. Fontana ${ }^{10}$, F. Fontanelli ${ }^{19, i}$, R. Forty ${ }^{35}$, O. Francisco ${ }^{2}$, M. Frank ${ }^{35}$, C. Frei ${ }^{35}$, M. Frosini ${ }^{17, f}$, S. Furcas ${ }^{20}$, A. Gallas Torreira ${ }^{34}$, D. Galli ${ }^{14, c}$, M. Gandelman ${ }^{2}$, P. Gandini ${ }^{52}$, Y. Gao ${ }^{3}$, J-C. Garnier ${ }^{35}$, J. Garofoli ${ }^{53}$, J. Garra Tico ${ }^{44}$ L. Garrido ${ }^{33}$, D. Gascon ${ }^{33}$, C. Gaspar ${ }^{35}$, R. Gauld ${ }^{52}$, N. Gauvin ${ }^{36}$, M. Gersabeck ${ }^{35}$, T. Gershon ${ }^{45,35}$, Ph. Ghez ${ }^{4}$, V. Gibson ${ }^{44}$, V.V. Gligorov ${ }^{35}$, C. Göbel ${ }^{54}$, D. Golubkov ${ }^{28}$, A. Golutvin ${ }^{50,28,35}$, A. Gomes ${ }^{2}$, H. Gordon ${ }^{52}$, M. Grabalosa Gándara ${ }^{33}$, R. Graciani Diaz ${ }^{33}$, L.A. Granado Cardoso ${ }^{35}$, E. Graugés ${ }^{33}$, G. Graziani ${ }^{17}$, A. Grecu ${ }^{26}$, E. Greening ${ }^{52}$, S. Gregson ${ }^{44}$, B. Gui ${ }^{53}$, E. Gushchin ${ }^{30}$, Yu. Guz ${ }^{32}$, T. Gys ${ }^{35}$, C. Hadjivasiliou ${ }^{53}$, G. Haefeli ${ }^{36}$, C. Haen ${ }^{35}$, S.C. Haines ${ }^{44}$, T. Hampson ${ }^{43}$, S. Hansmann-Menzemer ${ }^{11}$, R. Harji ${ }^{50}$, N. Harnew ${ }^{52}$, J. Harrison ${ }^{51}$, P.F. Harrison ${ }^{45}$, T. Hartmann ${ }^{55}$, J. He ${ }^{7}$, V. Heijne ${ }^{38}$, K. Hennessy ${ }^{49}$, P. Henrard ${ }^{5}$, J.A. Hernando Morata ${ }^{34}$, E. van Herwijnen ${ }^{35}$, E. Hicks ${ }^{49}$, K. Holubyev ${ }^{11}$, 
P. Hopchev ${ }^{4}$, W. Hulsbergen ${ }^{38}$, P. Hunt ${ }^{52}$, T. Huse ${ }^{49}$, R.S. Huston ${ }^{12}$, D. Hutchcroft ${ }^{49}$, D. Hynds ${ }^{48}$, V. Iakovenko ${ }^{41}$, P. Ilten ${ }^{12}$, J. Imong ${ }^{43}$, R. Jacobsson ${ }^{35}$, A. Jaeger ${ }^{11}$, M. Jahjah Hussein ${ }^{5}$, E. Jans ${ }^{38}$, F. Jansen ${ }^{38}$, P. Jaton ${ }^{36}$, B. Jean-Marie ${ }^{7}$, F. Jing ${ }^{3}$, M. John ${ }^{52}$, D. Johnson ${ }^{52}$, C.R. Jones ${ }^{44}$, B. $\mathrm{Jost}^{35}$, M. Kaballo ${ }^{9}$, S. Kandybei ${ }^{40}$, M. Karacson ${ }^{35}$, T.M. Karbach ${ }^{9}$, J. Keaveney ${ }^{12}$, I.R. Kenyon ${ }^{42}$, U. Kerzel ${ }^{35}$, T. Ketel ${ }^{39}$, A. Keune ${ }^{36}$, B. Khanji ${ }^{6}$, Y.M. Kim ${ }^{47}$, M. Knecht ${ }^{36}$, R.F. Koopman ${ }^{39}$, P. Koppenburg ${ }^{38}$, M. Korolev ${ }^{29}$, A. Kozlinskiy ${ }^{38}$, L. Kravchuk ${ }^{30}$, K. Kreplin ${ }^{11}$, M. Kreps ${ }^{45}$, G. Krocker ${ }^{11}$, P. Krokovny ${ }^{11}$, F. Kruse ${ }^{9}$, K. Kruzelecki ${ }^{35}$, M. Kucharczyk ${ }^{20,23,35, j}$, V. Kudryavtsev ${ }^{31}$, T. Kvaratskheliya ${ }^{28,35}$, V.N. La Thi ${ }^{36}$, D. Lacarrere ${ }^{35}$, G. Lafferty ${ }^{51}$, A. Lai ${ }^{15}$, D. Lambert ${ }^{47}$, R.W. Lambert ${ }^{39}$, E. Lanciotti ${ }^{35}$, G. Lanfranchi ${ }^{18}$, C. Langenbruch ${ }^{11}$,

T. Latham ${ }^{45}$, C. Lazzeroni ${ }^{42}$, R. Le Gac ${ }^{6}$, J. van Leerdam ${ }^{38}$, J.-P. Lees ${ }^{4}$, R. Lefèvre ${ }^{5}$, A. Leflat ${ }^{29,35}$, J. Lefrançois ${ }^{7}$, O. Leroy ${ }^{6}$, T. Lesiak ${ }^{23}$, L. Li ${ }^{3}$, L. Li Gioi ${ }^{5}$, M. Lieng 9 , M. $\operatorname{Liles}^{49}$, R. Lindner ${ }^{35}$, C. Linn ${ }^{11}$, B. Liu ${ }^{3}$, G. Liu ${ }^{35}$, J. von Loeben ${ }^{20}$, J.H. Lopes ${ }^{2}$, E. Lopez Asamar ${ }^{33}$, N. Lopez-March ${ }^{36}$, H. Lu ${ }^{3}$, J. Luisier ${ }^{36}$, A. Mac Raighne ${ }^{48}$, F. Machefert ${ }^{7}$, I.V. Machikhiliyan ${ }^{4,28}$, F. Maciuc ${ }^{10}$, O. Maev ${ }^{27,35}$, J. Magnin ${ }^{1}$, S. Malde ${ }^{52}$, R.M.D. Mamunur ${ }^{35}$, G. Manca ${ }^{15, d}$, G. Mancinelli ${ }^{6}$, N. Mangiafave ${ }^{44}$, U. Marconi ${ }^{14}$, R. Märki ${ }^{36}$, J. Markss ${ }^{11}$, G. Martellottii ${ }^{22}$, A. Martens ${ }^{8}$, L. Martin ${ }^{52}$, A. Martín Sánchez ${ }^{7}$, M. Martinelli ${ }^{38}$, D. Martinez Santos ${ }^{35}$, A. Massafferri ${ }^{1}$, Z. Mathe ${ }^{12}$, C. Matteuzzi ${ }^{20}$, M. Matveev ${ }^{27}$, E. Maurice $^{6}$, B. Maynard ${ }^{53}$, A. Mazurov ${ }^{16,30,35}$, G. McGregor ${ }^{51}$, R. McNulty ${ }^{12}$, M. Meissner ${ }^{11}$, M. Merk ${ }^{38}$, J. Merkel ${ }^{9}$, S. Miglioranzi ${ }^{35}$, D.A. Milanes ${ }^{13}$, M.-N. Minard ${ }^{4}$, J. Molina Rodriguez ${ }^{54}$, S. Monteil ${ }^{5}$, D. Moran ${ }^{12}$, P. Morawski ${ }^{23}$, R. Mountain ${ }^{53}$, I. Mous ${ }^{38}$, F. Muheim ${ }^{47}$, K. Müller ${ }^{37}$, R. Muresan ${ }^{26}$, B. Muryn ${ }^{24}$, B. Muster ${ }^{36}$, J. Mylroie-Smith ${ }^{49}$, P. Naik ${ }^{43}$, T. Nakada ${ }^{36}$, R. Nandakumar ${ }^{46}$, I. Nasteva ${ }^{1}$, M. Needham ${ }^{47}$, N. Neufeld ${ }^{35}$, A.D. Nguyen ${ }^{36}$, C. Nguyen-Mau ${ }^{36, o}$, M. Nicol ${ }^{7}, \mathrm{~V}_{\text {. Niess }}{ }^{5}$, N. Nikitin ${ }^{29}$, A. Nomerotski ${ }^{52,35}$, A. Novoselov ${ }^{32}$, A. Oblakowska-Mucha ${ }^{24}$, V. Obraztsov ${ }^{32}$, S. Oggero $^{38}$, S. Ogilvy ${ }^{48}$, O. Okhrimenko ${ }^{41}$, R. Oldeman ${ }^{15, d, 35}$, M. Orlandea ${ }^{26}$, J.M. Otalora Goicochea ${ }^{2}$, P. Owen ${ }^{50}$, B.K. Pal ${ }^{53}$, J. Palacios ${ }^{37}$, A. Palano ${ }^{13, b}$, M. Palutan ${ }^{18}$, J. Panman ${ }^{35}$, A. Papanestis ${ }^{46}$, M. Pappagallo ${ }^{48}$, C. Parkes ${ }^{51}$, C.J. Parkinson ${ }^{50}$, G. Passaleva ${ }^{17}$, G.D. Patel ${ }^{49}$, M. Patel ${ }^{50}$, S.K. Paterson ${ }^{50}$, G.N. Patrick ${ }^{46}$, C. Patrignani ${ }^{19, i}$, C. Pavel-Nicorescu ${ }^{26}$, A. Pazos Alvarez ${ }^{34}$, A. Pellegrino ${ }^{38}$, G. Penso ${ }^{22, l}$, M. Pepe Altarelli $^{35}$, S. Perazzini ${ }^{14, c}$, D.L. Perego ${ }^{20, j}$, E. Perez Trigo ${ }^{34}$, A. Pérez-Calero Yzquierdo ${ }^{33}$, P. Perret $^{5}$, M. Perrin-Terrin ${ }^{6}$, G. Pessina ${ }^{20}$, A. Petrolini ${ }^{19, i}$, A. Phan ${ }^{53}$, E. Picatoste Olloqui ${ }^{33}$, B. Pie Valls ${ }^{33}$, B. Pietrzyk ${ }^{4}$, T. Pilar̆ ${ }^{45}$, D. Pinci ${ }^{22}$, R. Plackett ${ }^{48}$, S. Playfer ${ }^{47}$, M. Plo Casasus ${ }^{34}$, G. Polok ${ }^{23}$, A. Poluektov ${ }^{45,31}$, E. Polycarpo ${ }^{2}$, D. Popov ${ }^{10}$, B. Popovici ${ }^{26}$, C. Potterat ${ }^{33}$, A. Powell ${ }^{52}$, J. Prisciandaro ${ }^{36}$, V. Pugatch ${ }^{41}$, A. Puig Navarro ${ }^{33}$, W. $\operatorname{Qian}^{53}$, J.H. Rademacker ${ }^{43}$, B. Rakotomiaramanana ${ }^{36}$, M.S. Rangel ${ }^{2}$, I. Raniuk ${ }^{40}$, G. Raven $^{39}$, S. Redford ${ }^{52}$, M.M. Reid ${ }^{45}$, A.C. dos Reis ${ }^{1}$, S. Ricciardi ${ }^{46}$, A. Richards ${ }^{50}$, K. Rinnert ${ }^{49}$, D.A. Roa Romero ${ }^{5}$, P. Robbe ${ }^{7}$, E. Rodrigues ${ }^{48,51}$, F. Rodrigues ${ }^{2}$, P. Rodriguez Perez ${ }^{34}$, G.J. Rogers ${ }^{44}$, S. Roiser ${ }^{35}$, V. Romanovsky ${ }^{32}$, M. Rosello ${ }^{33, n}$, J. Rouvinet ${ }^{36}$, T. Ruf ${ }^{35}$, H. Ruiz ${ }^{33}$, G. Sabatino ${ }^{21, k}$, J.J. Saborido Silva ${ }^{34}$, N. Sagidova ${ }^{27}$, P. Sail ${ }^{48}$, B. Saitta ${ }^{15, d}$, C. Salzmann ${ }^{37}$, M. Sannino ${ }^{19, i}$, R. Santacesaria ${ }^{22}$, C. Santamarina Rios $^{34}$, R. Santinelli ${ }^{35}$, E. Santovetti ${ }^{21, k}$, M. Sapunov ${ }^{6}$, A. Sarti ${ }^{18, l}$, C. Satriano ${ }^{22, m}$, A. Satta ${ }^{21}$, M. Savrie ${ }^{16, e}$, D. Savrina ${ }^{28}$, P. Schaack ${ }^{50}$, M. Schiller ${ }^{39}$, 
H. Schindler ${ }^{35}$, S. Schleich ${ }^{9}$, M. Schlupp ${ }^{9}$, M. Schmelling ${ }^{10}$, B. Schmidt ${ }^{35}$, O. Schneider ${ }^{36}$, A. Schopper ${ }^{35}$, M.-H. Schune ${ }^{7}$, R. Schwemmer ${ }^{35}$, B. Sciascia ${ }^{18}$, A. Sciubba ${ }^{18, l}$, M. $\operatorname{Seco}^{34}$, A. Semennikov ${ }^{28}$, K. Senderowska ${ }^{24}$, I. Sepp ${ }^{50}$, N. Serra ${ }^{37}$, J. Serrano ${ }^{6}$, P. Seyfert ${ }^{11}$, M. Shapkin ${ }^{32}$, I. Shapoval ${ }^{40,35}$, P. Shatalov ${ }^{28}$, Y. Shcheglov ${ }^{27}$, T. Shears ${ }^{49}$, L. Shekhtman ${ }^{31}$, O. Shevchenko ${ }^{40}$, V. Shevchenko ${ }^{28}$, A. Shires ${ }^{50}$, R. Silva Coutinho ${ }^{45}$, T. Skwarnicki ${ }^{53}$, N.A. Smith ${ }^{49}$, E. Smith ${ }^{52,46}$, K. Sobczak ${ }^{5}$, F.J.P. Soler ${ }^{48}$, A. Solomin ${ }^{43}$, F. Soomro ${ }^{18,35}$, B. Souza De Paula $^{2}$, B. $\operatorname{Spaan}^{9}$, A. Sparkes ${ }^{47}$, P. Spradlin ${ }^{48}$, F. Stagni ${ }^{35}$, S. Stahl ${ }^{11}$, O. Steinkamp ${ }^{37}$, S. Stoica ${ }^{26}$, S. Stone ${ }^{53,35}$, B. Storaci ${ }^{38}$, M. Straticiuc ${ }^{26}$, U. Straumann ${ }^{37}$, V.K. Subbiah ${ }^{35}$, S. Swientek ${ }^{9}$, M. Szczekowski ${ }^{25}$, P. Szczypka ${ }^{36}$, T. Szumlak ${ }^{24}$, S. T'Jampens ${ }^{4}$, E. Teodorescu ${ }^{26}$, F. Teubert ${ }^{35}$, C. Thomas ${ }^{52}$, E. Thomas ${ }^{35}$, J. van Tilburg ${ }^{11}$, V. Tisserand ${ }^{4}$, M. Tobin ${ }^{37}$, S. Topp-Joergensen ${ }^{52}$, N. Torr ${ }^{52}$, E. Tournefier ${ }^{4,50}$, S. Tourneur ${ }^{36}$, M.T. Tran ${ }^{36}$, A. Tsaregorodtsev ${ }^{6}$, N. Tuning ${ }^{38}$, M. Ubeda Garcia ${ }^{35}$, A. Ukleja ${ }^{25}$, U. Uwer ${ }^{11}$, V. Vagnoni ${ }^{14}$, G. Valenti ${ }^{14}$, R. Vazquez Gomez ${ }^{33}$, P. Vazquez Regueiro ${ }^{34}$, S. Vecchi ${ }^{16}$, J.J. Velthuis ${ }^{43}$, M. Veltri ${ }^{17, g}$, B. Viaud ${ }^{7}$, I. Videau ${ }^{7}$, D. Vieira ${ }^{2}$, X. Vilasis-Cardona ${ }^{33, n}$, J. Visniakov ${ }^{34}$, A. Vollhardt ${ }^{37}$, D. Volyanskyy ${ }^{10}$, D. Voong ${ }^{43}$, A. Vorobyev ${ }^{27}, \mathrm{H}$. $\operatorname{Voss}^{10}$, R. Waldi ${ }^{55}$, S. Wandernoth ${ }^{11}$, J. Wang ${ }^{53}$, D.R. Ward ${ }^{44}$, N.K. Watson ${ }^{42}$, A.D. Webber ${ }^{51}$, D. Websdale ${ }^{50}$, M. Whitehead ${ }^{45}$, D. Wiedner ${ }^{11}$, L. Wiggers ${ }^{38}$, G. Wilkinson ${ }^{52}$, M.P. Williams ${ }^{45,46}$, M. Williams ${ }^{50}$, F.F. Wilson ${ }^{46}$, J. Wishahi ${ }^{9}$, M. Witek ${ }^{23}$, W. Witzeling ${ }^{35}$, S.A. Wotton ${ }^{44}$, K. Wyllie ${ }^{35}$, Y. Xie ${ }^{47}$, F. Xing ${ }^{52}$, Z. Xing ${ }^{53}$, Z. Yang ${ }^{3}$, R. Young ${ }^{47}$, O. Yushchenko ${ }^{32}$, M. Zangoli ${ }^{14}$, M. Zavertyaev ${ }^{10, a}$, F. Zhang ${ }^{3}$, L. Zhang ${ }^{53}$, W.C. Zhang ${ }^{12}$, Y. Zhang ${ }^{3}$, A. Zhelezov ${ }^{11}$, L. Zhong ${ }^{3}$, A. Zvyagin ${ }^{35}$.

1: Centro Brasileiro de Pesquisas Físicas (CBPF), Rio de Janeiro, Brazil

2: Universidade Federal do Rio de Janeiro (UFRJ), Rio de Janeiro, Brazil

3: Center for High Energy Physics, Tsinghua University, Beijing, China

4: LAPP, Université de Savoie, CNRS/IN2P3, Annecy-Le-Vieux, France

5: Clermont Université, Université Blaise Pascal, CNRS/IN2P3, LPC, Clermont-Ferrand, France

6: CPPM, Aix-Marseille Université, CNRS/IN2P3, Marseille, France

7: LAL, Université Paris-Sud, CNRS/IN2P3, Orsay, France

8: LPNHE, Université Pierre et Marie Curie, Université Paris Diderot, CNRS/IN2P3, Paris, France

${ }^{9}$ : Fakultät Physik, Technische Universität Dortmund, Dortmund, Germany

10: Max-Planck-Institut für Kernphysik (MPIK), Heidelberg, Germany

11: Physikalisches Institut, Ruprecht-Karls-Universität Heidelberg, Heidelberg, Germany

12: School of Physics, University College Dublin, Dublin, Ireland

13: Sezione INFN di Bari, Bari, Italy

14: Sezione INFN di Bologna, Bologna, Italy

15: Sezione INFN di Cagliari, Cagliari, Italy

16: Sezione INFN di Ferrara, Ferrara, Italy

17: Sezione INFN di Firenze, Firenze, Italy

18: Laboratori Nazionali dell'INFN di Frascati, Frascati, Italy

19: Sezione INFN di Genova, Genova, Italy

${ }^{20}$ : Sezione INFN di Milano Bicocca, Milano, Italy 
21: Sezione INFN di Roma Tor Vergata, Roma, Italy

22: Sezione INFN di Roma La Sapienza, Roma, Italy

23: Henryk Niewodniczanski Institute of Nuclear Physics Polish Academy of Sciences, Kraków, Poland

24: AGH University of Science and Technology, Kraków, Poland

25: Soltan Institute for Nuclear Studies, Warsaw, Poland

26: Horia Hulubei National Institute of Physics and Nuclear Engineering, Bucharest-Magurele, Romania

27: Petersburg Nuclear Physics Institute (PNPI), Gatchina, Russia

28: Institute of Theoretical and Experimental Physics (ITEP), Moscow, Russia

29: Institute of Nuclear Physics, Moscow State University (SINP MSU), Moscow, Russia

30: Institute for Nuclear Research of the Russian Academy of Sciences (INR RAN), Moscow, Russia

31: Budker Institute of Nuclear Physics (SB RAS) and Novosibirsk State University, Novosibirsk, Russia

32: Institute for High Energy Physics (IHEP), Protvino, Russia

33: Universitat de Barcelona, Barcelona, Spain

34: Universidad de Santiago de Compostela, Santiago de Compostela, Spain

35: European Organization for Nuclear Research (CERN), Geneva, Switzerland

36: Ecole Polytechnique Fédérale de Lausanne (EPFL), Lausanne, Switzerland

37: Physik-Institut, Universität Zürich, Zürich, Switzerland

38: Nikhef National Institute for Subatomic Physics, Amsterdam, The Netherlands

39: Nikhef National Institute for Subatomic Physics and VU University Amsterdam, Amsterdam, The Netherlands

40: NSC Kharkiv Institute of Physics and Technology (NSC KIPT), Kharkiv, Ukraine

41: Institute for Nuclear Research of the National Academy of Sciences (KINR), Kyiv, Ukraine

42: University of Birmingham, Birmingham, United Kingdom

${ }^{43}$ : H.H. Wills Physics Laboratory, University of Bristol, Bristol, United Kingdom

${ }^{44}$ : Cavendish Laboratory, University of Cambridge, Cambridge, United Kingdom

45: Department of Physics, University of Warwick, Coventry, United Kingdom

46: STFC Rutherford Appleton Laboratory, Didcot, United Kingdom

47: School of Physics and Astronomy, University of Edinburgh, Edinburgh, United Kingdom

48. School of Physics and Astronomy, University of Glasgow, Glasgow, United Kingdom

${ }^{49}$ : Oliver Lodge Laboratory, University of Liverpool, Liverpool, United Kingdom

50: Imperial College London, London, United Kingdom

51: School of Physics and Astronomy, University of Manchester, Manchester, United Kingdom

52: Department of Physics, University of Oxford, Oxford, United Kingdom

53: Syracuse University, Syracuse, NY, United States

54: Pontifícia Universidade Católica do Rio de Janeiro (PUC-Rio), Rio de Janeiro, Brazil, associated to ${ }^{2}$

${ }^{55}$ : Physikalisches Institut, Universität Rostock, Rostock, Germany, associated to ${ }^{11}$

${ }^{a}$ : P.N. Lebedev Physical Institute, Russian Academy of Science (LPI RAS), Moscow, Russia

${ }^{b}$ : Università di Bari, Bari, Italy

c: Università di Bologna, Bologna, Italy

${ }^{d}$ : Università di Cagliari, Cagliari, Italy

$e^{e}$ : Università di Ferrara, Ferrara, Italy

$f^{f}$ : Università di Firenze, Firenze, Italy 
g: Università di Urbino, Urbino, Italy

${ }^{h}$ : Università di Modena e Reggio Emilia, Modena, Italy

${ }^{i}$ : Università di Genova, Genova, Italy

${ }^{j}$ : Università di Milano Bicocca, Milano, Italy

${ }^{k}$ : Università di Roma Tor Vergata, Roma, Italy

l: Università di Roma La Sapienza, Roma, Italy

${ }^{m}$ : Università della Basilicata, Potenza, Italy

${ }^{n}$ : LIFAELS, La Salle, Universitat Ramon Llull, Barcelona, Spain

${ }^{o}$ : Hanoi University of Science, Hanoi, Viet Nam 